\title{
A new perspective on the pleiotropic blood pressure improvement effect of sitagliptin: downregulation of miRNA-155 and miRNA-21
}

\author{
Yilmaz-Bozoglan $\mathrm{M}^{1}$, Gul-Kahraman $\mathrm{K}^{1}$, Serhatlioglu I², Onalan $\mathrm{E}^{3}$, Akkopru $\mathrm{MC}^{1}$, Sahna E \\ Department of Pharmacology, Faculty of Medicine, Firat University, Elazig Turkey. \\ mervehcttp@hotmail.com
}

\begin{abstract}
INTRODUCTION: Deterioration of vascular responses is the crucial event in the initiation of cardiovascular problems in hypertension (HT) and diabetes mellitus (DM). A well-known oral antidiabetic, sitagliptin, has pleiotropic effects besides improving glycemic state in type-2 DM. This study aimed to investigate the therapeutic effect of sitagliptin on blood pressure with previously unassessed parameters of well-known pathophysiological processes and especially at the microRNA (miRNA) level where there are many unknowns. METHODS: N-nitro-L-arginine methyl ester (L-NAME)-induced HT model was performed on nondiabetic male rats. Four groups (including 7 rats in each) were formed: normotensives, sitagliptin-treated, HT and sitagliptintreated HT. Asymmetric dimethylarginine (ADMA), intercellular adhesion molecule-1 (ICAM-1) and tyrosine hydroxylase (TH), HT related miRNAs were evaluated. In-vitro vessel responses were observed. RESULTS: L-NAME led to a significant increase in blood pressure. Hypertensives exhibited significantly increased contractile responses, consistent with increased ADMA, ICAM-1. Sitagliptin decreased TH levels but not statistically significantly. The new side of the study was the miRNA-21 and miRNA-155 expressions were in line with other parameters in both the HT and sitagliptin-treated HT groups.

CONCLUSION: Sitagliptin may control comorbidities, especially HT and introduces new targets to alleviate vascular responses. The new knowledge is; sitagliptin may show these effects through microRNAs (Tab. 2, Fig. 6, Ref. 46). Text in PDF www.elis.sk KEY WORDS: endothelial dysfunction, inflammation, miRNA-21, miRNA-155, sitagliptin, blood pressure.
\end{abstract}

\section{Introduction}

Despite all researches and the giant antihypertensive drug market, 9.4 million people die every year due to hypertension (HT) and its complications. By 2025 approximately $1 / 3$ of the world adult population will be diagnosed with HT $(1,2)$. Diabetes mellitus (DM) is the most common well-known comorbidity that accompanies HT (3). Last guidelines reported that every $10-20 \mathrm{mmHg}$ increase in systolic blood pressure (SBP) doubles the cardiovascular risk (4). Endothelial dysfunction, increased sympathetic activity and inflammation are crucial points of the pathogenesis of HT and DM (5). Therefore, if an antidiabetic drug improves these

${ }^{1}$ Department of Pharmacology, Faculty of Medicine, Firat University, Elazig, Turkey, ${ }^{2}$ Department of Biophysics, Faculty of Medicine, Firat University, Elazig, Turkey, and ${ }^{3}$ Department of Medical Biology, Faculty of Medicine, Firat University, Elazig, Turkey

Address for correspondence: M. Yilmaz Bozoglan, MD, Assoc. Prof., Department of Pharmacology, Faculty of Medicine, Firat University, 23119, Elazig, Turkey.

Phone: +90.5446927371 , Fax: +90.4242379138

Acknowledgements: The work was done in the Firat University School of Medicine and supported by Firat University Scientific Research Project Department (TF 17.32). processes in addition to intensive glucose control, it will reduce cardiovascular risk (6).

Sitagliptin is well-tolerated oral antidiabetic which inhibits the dipeptidyl-peptidase-4 (DPP-4) that breaks down incretins (7). Since incretins are released in response to carbohydrate intake, sitagliptin usually does not cause hypoglycemia. Administration once daily without dependence on hunger or satiety, weight control with the reduction of appetite, lower gastrointestinal side effects than other oral antidiabetics are the known advantages of sitagliptin. Moreover, widespread expression of DPP-4 in the vascular bed increases the possibility of its role in vascular function (8). Some studies support the opinion that sitagliptin has a pleiotropic property that lowers blood pressure (BP) through one or more pathways that are not very clear. The most common opinion is that sitagliptin activates endothelial nitric oxide synthase (eNOS) and increases nitric oxide (NO) bioavailability that improves the disrupted balance of endothelium $(9,10)$. Infiltration of adhesion molecules into the arterial wall and a low level of inflammation is another initial step in HT (1). The intercellular adhesion molecule-1 (ICAM-1) has been implicated in the recruitment and adhesion of leukocytes in several cardiac diseases (12). It is known that proinflammatory cytokines (TNF- $\alpha$, IL-6, CRP and CCL2) and some adhesion molecules were decreased with sitagliptin treatment (13, 
14). However, the effect of sitagliptin on ICAM-1 levels in hypertension was not evaluated in any experimental or clinical study.

On the other hand, our literature review showed that the most recent studies on sitagliptin are now also related to COVID-19. We have learned that DM and HT are the most common accompanying diseases in severe COVID-19 (2). And sitagliptin, an effective plasma glucose-lowering drug, seems to be important even in this critical disease with its main or pleiotropic characteristics $(16,17)$.

MicroRNAs (miRNAs) are single-stranded, non-coding genes that impact the expression of hundreds of mRNA by silencing them and many of them are being discovered to be disease-specific (18). miRNA-155 is a critical one that may be modulating the HT through suppressing excessive eNOS expression and inflammatory process $(19,20)$. miRNA-21 is one of the most intensively investigated miRNAs in the context of the cardiovascular system and is the most prominent non-coding gene associated with HT (21, $22,23)$. According to our research, the effect of sitagliptin on hypertension has not been previously evaluated at the miRNA level.

Here, through miRNA-155 and miRNA-21, we have further unveiled the effect of sitagliptin in hypertension as well as the well-known pathophysiological processes with previously unassessed parameters. We performed an experimental HT model on non-diabetic rats and compared the consistency of the BP measurements with the results of the endothelial contraction-relaxation responses, serum levels of asymmetric dimethylarginine (ADMA) which is an eNOS inhibitor that reflects endothelial dysfunction, tyrosine hydroxylase (TH) is the key enzyme of catecholamine synthesis in the sympathetic system, and ICAM-1 as an inflammatory biomarker.

\section{Methods and materials}

\section{Animals and experimental design}

The experiments have been carried out in accordance with The Code of Ethics of the World Medical Association (Declara-

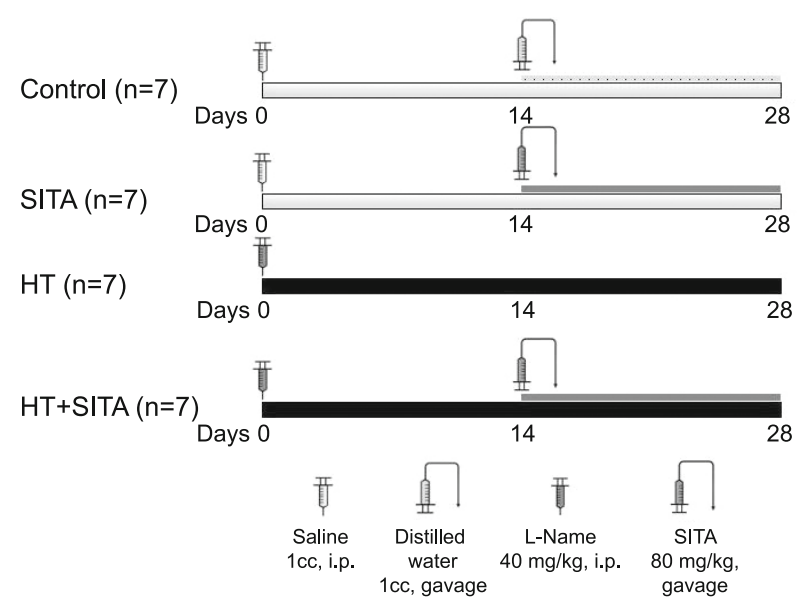

Fig. 1. Illustration of experimental protocols. SITA - sitagliptin; HT - hypertension; L-NAME - N-nitro-L-arginine methyl ester; i.p. intraperitoneal, tion of Helsinki). Necessary approvals for the experiments were confirmed by the Local Ethics Committee of the Firat University Animal Experiments Center (Approval number: 133) and all investigations adhere to the ARRIVE2 Guidelines. Twenty-eight 10-weeks-old male Sprague-Dawley rats, weighing 240-260 g, were obtained from Firat University Animal Experiments Center (Elazig, Turkey) and held in cages with standard chow and water ad libitum, $24 \pm 2{ }^{\circ} \mathrm{C}$ and $40-60 \%$ humidity under 12 hours light and dark cycles. Rats were randomly divided into 4 groups with 7 rats in each one (Random numbers were generated in Microsoft Excel. (i) The Control group reflected normotensives. Only $1 \mathrm{cc} /$ day saline for 4 weeks and distilled water by gavage between the 14 th and 28th days, were given. (ii) The Sitagliptin group was administered $1 \mathrm{cc} /$ day saline for 28 days and sitagliptin for 14 days (between the 14th and 28th days). (iii) HT group was injected LNAME, starting from the first day to the end of the experiment. (iv) HT + Sitagliptin group was administered L-NAME as in HT group and sitagliptin in last 14 days (Fig. 1).

\section{Drugs and chemicals}

L-NAME from Cayman Chemical Company (Batch: 049195812), Sitagliptin from Toronto Research Chemicals Inc. Canada (lot number: 15-SCC-61-1), phenylephrine (Phe) and acetylcholine (ACh) (Acros Organics, New Jersey, USA), and sodium nitroprusside (SNP) from Alfa Aesar, Germany (lot number: 10201564) were purchased from respective vendors. HT was induced by intraperitoneal injection of $40 \mathrm{mg} / \mathrm{kg} /$ day L-NAME, a NOS inhibitor, dissolved in $1 \mathrm{cc}$ of $0.9 \%$ saline. $80 \mathrm{mg} / \mathrm{kg} /$ day dose of sitagliptin that dissolved in distilled water was given by oral gavage. The dosage was adjusted according to Giannaco's study since chronic administration of sitagliptin with this dose inhibits DPP-4 activity by $85 \%$ and does not cause hypoglycemia (3). Fresh solutions were prepared from powdered chemicals every day and applied at the same times of the days.

\section{In-vivo studies}

BP measurements: Indirect BP Recorder (MAY BPHR 9610PC TAIL-CUFF) and MP-36, Biopac, transducer were used for recording the non-invasive measurements. Rats waited approximately 25-30 min. to stabilize. Then SBPs were measured three times during the experiment; at the beginning, 14th day and the last one was before decapitation. BPs that were used in statistics were the means of five times recordings. Bodyweight measurements: Rats, initially approximately 250 grams, were weighed three times during the experiment, on the days of BP measurements. Plasma glucose levels: Random measurements from femoral vein blood by a glucometer were done on distinctly selected rats from each group and no hypoglycemic measurement was observed (data not shown).

\section{In-vitro studies}

Isolation of vessels: Just after decapitation, the thoracic aorta from the arch down to the diaphragm was isolated and cleaned from fat and connective tissue gently. The buffer Krebs Henseleit at pH: 7.4 was used while cleaning and for organ bath, as in Tang and colleagues' study (4). Approximately $4 \mathrm{~mm}$ long aortic rings 
Tab. 1 Body weights in chronically nitric oxide synthase inhibited rats and in hypertensive rats after 14 days of sitagliptin treatment.

\begin{tabular}{lccc}
\hline Groups & Day 0 $(\mathrm{g})$ & Day 14 $(\mathrm{g})$ & Day 28 $(\mathrm{g})$ \\
\hline Control & $245.61 \pm 3.15$ & $255.30 \pm 8.41$ & $273.21 \pm 12.50^{\#}$ \\
SITA & $247.34 \pm 2.28$ & $265.44 \pm 5.81$ & $271.30 \pm 5.76$ \\
HT & $244.24 \pm 2.66$ & $260.76 \pm 3.19$ & $275.45 \pm 3.60^{\#}$ \\
HT+SITA & $250.07 \pm 1.89$ & $271.29 \pm 5.70$ & $280.47 \pm 10.72$ \\
\hline
\end{tabular}

Data are presented as mean \pm S.E.M. \#. $\mathrm{p}<0.05$ compared to day 14 . SITA - sitagliptin; HT - hypertension
Tab. 2 Contraction to Phe and relaxation to $\mathrm{ACh}$ (expressed as $\mathrm{EC}_{50}$ ) in thoracic aorta of L-NAME administered and sitagliptin treated groups.

\begin{tabular}{lcccc}
\hline$-\log \mathrm{EC}_{50}(\mu \mathrm{M})$ & Control & SITA & HT & HT + SITA \\
\hline Phe & $6.47 \pm 0.05^{\mathrm{c}}$ & $6.13 \pm 0.03^{\mathrm{c}}$ & $6.91 \pm 0.07$ & $5.91 \pm 0.06^{\text {abc }}$ \\
$\mathrm{ACh}$ & $7.22 \pm 0.04$ & $7.71 \pm 0.04$ & $6.48 \pm 0.06^{\mathrm{ab}}$ & $7.35 \pm 0.10^{\mathrm{c}}$ \\
\hline $\mathrm{n}=7$ for each group. Data are expressed as the mean \pm S.E.M. a. $\mathrm{p}<0.05$ compared \\
to control, b. $\mathrm{p}<0.05$ compared to SITA, c. $\mathrm{p}<0.05$ compared to HT. SITA - sita- \\
gliptin; $\mathrm{HT}$ - hypertension; L-NAME - N-nitro-L-arginine methyl ester; $\mathrm{EC}_{50}$ : molar \\
concentration at which the effect is half-maximal
\end{tabular}

concentration at which the effect is half-maximal

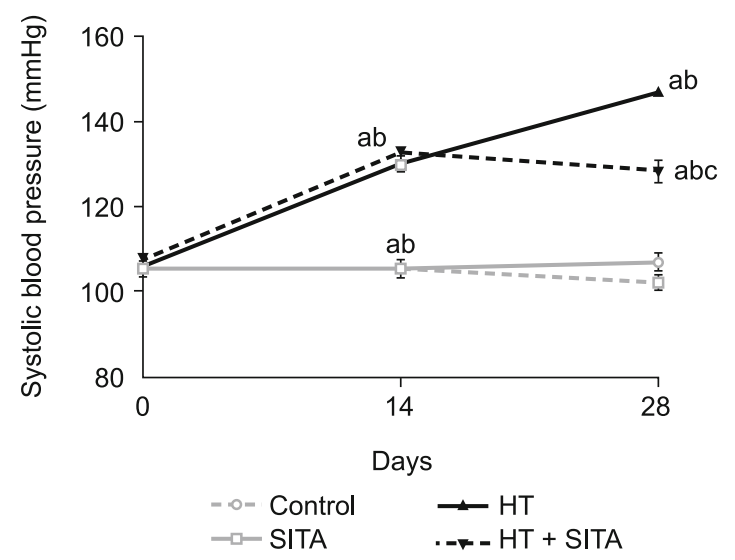

Fig. 2. Effect of sitagliptin on systolic blood pressure in L-NAME induced hypertension. Time course of systolic blood pressures in all groups for 4 weeks. $n=7$, for each group. Data are represented as Mean \pm S.E.M. a. $p<0.05$ compared to control, $b$. $p<0.05$ compared to SITA, c. $\mathrm{p}<0.05$ compared to HT. SITA - sitagliptin; HT - hypertension. connected on Force-Displacement Transducer in $20 \mathrm{~mL}$ temperature-controlled $\left(37{ }^{\circ} \mathrm{C}\right)$ and $95 \% \mathrm{O}_{2}$ and $5 \% \mathrm{CO}_{2}$ gas mixture aerated two baths. Isometric tension-relaxation measurements: Vessels were equilibrated for $60 \mathrm{~min}$ at $2.0 \mathrm{~g}$ resting tension, with changes of bathing fluid every $15 \mathrm{~min}$. After the equilibrium, the vessel rings were exposed to increasing concentration $\left(1 \times 10^{-9}-1 \times 10^{-4} \mathrm{M}\right)$ of Phe and cumulative vasoconstriction were seen in a concentration-dependent manner. Relaxation responses with ACh after a submaximal Phe precontraction were also measured. ANO donor, SNP was used to verify the integrity of smooth muscle in thoracic aortas in a cumulative fashion, too. At the end of the procedure $80 \mathrm{mmol} / \mathrm{L} \mathrm{KCl}$ was applied to confirm the viability of vessels via receptor-independent contraction. Contractile responses were measured as force $(\mathrm{g})$ and relaxation responses as percentage of forces. The molar concentration at which the effect is half-maximal is denoted $\mathrm{EC}_{50}$ whereas the maximal effect is $\mathrm{E}_{\max } . \mathrm{EC}_{50}$ values were determined with linear regression of the log concentration-response curve.

\section{Molecular analyses}

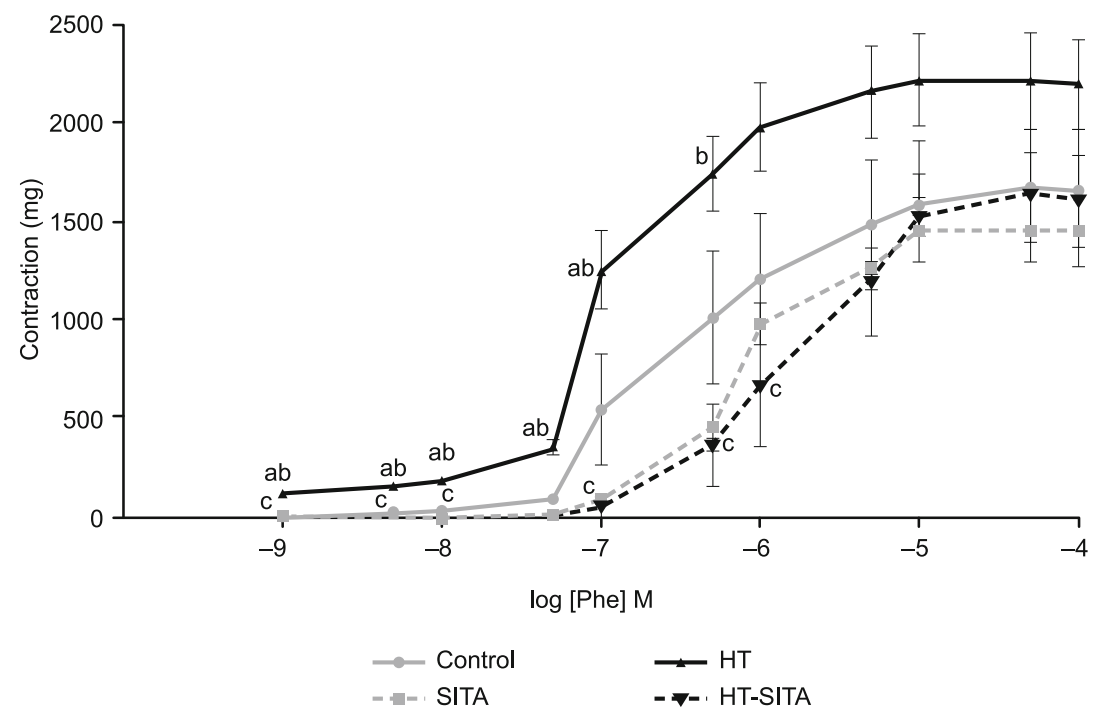

Fig. 3. Concentration-response curves for exogenous phenylephrine (Phe) expressed in milligrams. Statistical differences were analyzed between the tested groups at each concentration of the Phe. $n=7$ for each group. Data are expressed as the mean \pm S.E.M. a. $p<0.05$ compared to control, b. $p<0.05$ compared to SITA, c. $p<0.05$ compared to HT. SITA - sitagliptin; HT - hypertension.
Serum samples were obtained after centrifugation of the blood at $+4{ }^{\circ} \mathrm{C}$ and aortic tissues stored at $-80^{\circ} \mathrm{C}$ (Nuaire, Mexico).

Enzyme-linked immunosorbent assay (ELISA)

ELISA was employed to determine the serum levels of ADMA, ICAM-1, TH and supernatant of the rats. The examination of ADMA and ICAM-1 was carried out according to the instructions of the kit (FineTest, Wuhan, China, batch numbers: U256D010 and R0028D010). TH examined according to company protocol (Sunredbio, Shanghai, China, Ref. DZE SRB-T-84339).

Quantitative real-time polymerase chain reaction analysis ( $q R T-P C R)$

According to the manufacturer's protocol RNA isolation from blood was performed by the Trizol method (Tri Reagent Cat no: 15596026, Invitrogen, Carlsbad, CA). The tissue samples were homogenized with an automated homogenizer (Next Ad- 
vance, Troy, USA). Qubit ${ }^{\circledR}$ RNA Assay Kit with The Qubit 2.0 Fluorometer (Invitrogen/Molecular Probes) was used for spectrophotometric RNA measurement. To synthesize cDNA by reverse transcription (TaqMan MicroRNA Reverse Transcription Kit, Cat No: 4368814, Applied Biosystem, Foster City, CA), $5 \mu 1$ RNA was used. The qRT-PCR program consisted of 4 steps that each had a different temperature and duration, was performed in triplicate, to amplify the cDNA sequences.

\section{Statistical analysis}

Results were presented as means \pm S.E.M. One-way analysis of variance (ANOVA) followed by Tukey's post hoc test was used
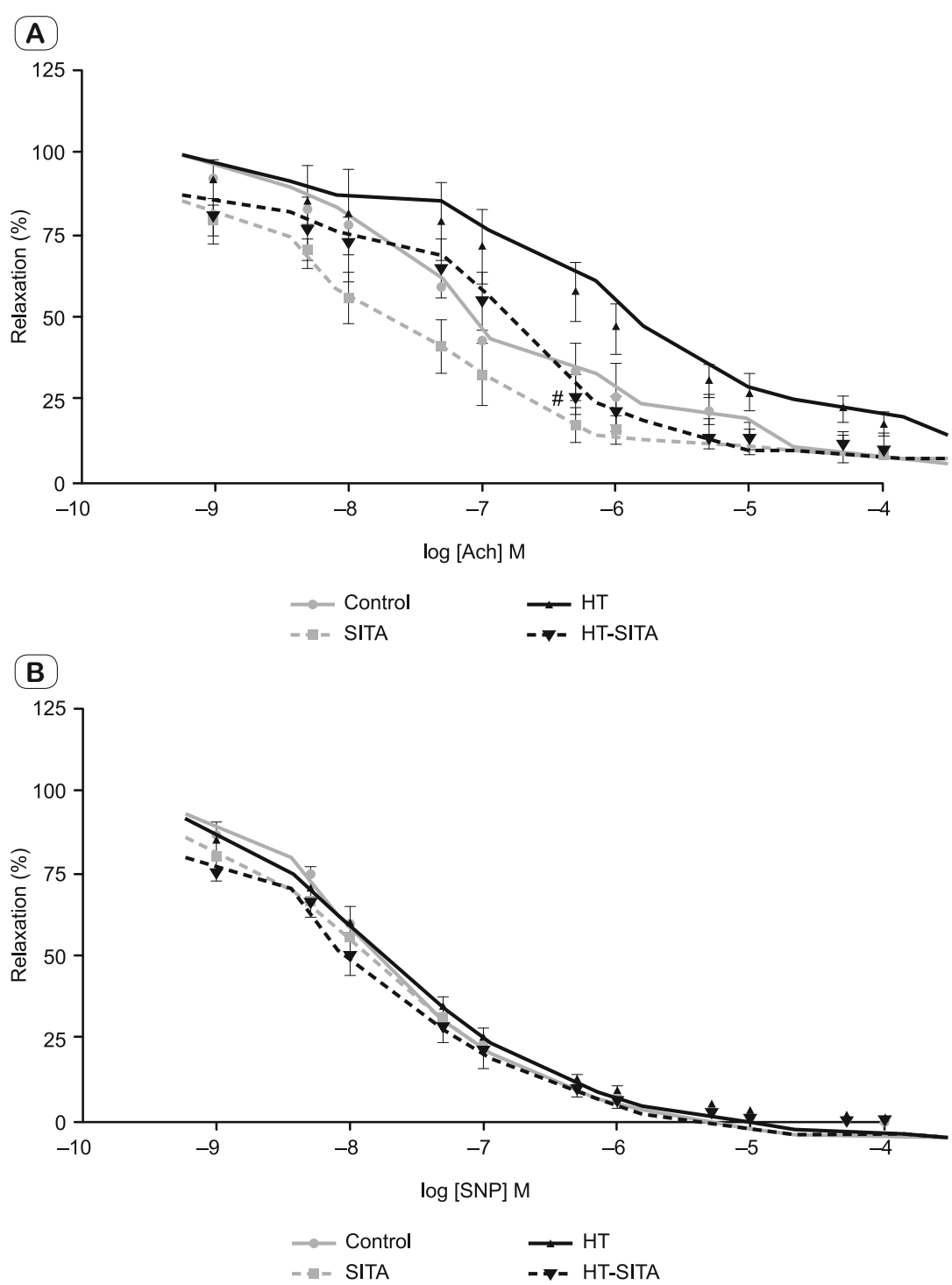

Fig. 4. Endothelium-dependent relaxation evoked by acetylcholine (ACh) (A) and endotheliumindependent relaxation evoked by sodium nitroprusside (SNP) (B) administration. The normalized concentration-response curves to $\mathrm{ACh}$ and SNP. Statistical differences were analyzed between the tested groups at each concentration of $\mathrm{ACh}$ and SNP. $\mathrm{n}=7$ for each group. Data are expressed as the mean \pm S.E.M. a. $p<0.05$ compared to control, $b$. $p<0.05$ compared to SITA, c. $p<0.05$ compared to HT. SITA - sitagliptin; HT - hypertension. to analyze the differences. The paired t-test was used to evaluate the difference between the values of the same group at different times. Nonlinear regression analysis was used in the computation of $\mathrm{EC}_{50}$ values. A p $<0.05$ was considered significant.

\section{Results}

Effects of sitagliptin on BPs and body weights

L-NAME significantly increased the SBPs progressively in 4 weeks. While SBP of HT group was $146.99 \pm 0.74 \mathrm{mmHg}$, sitagliptin treated HT group was $128.22 \pm 2.62 \mathrm{mmHg}$, on the 28 th day of the experiment. Sitagliptin application for 14 days reduced SBP, significantly ( $<<0.05$ ) (Fig. 2). Body weight changes during the experiment are shown in Table 1. There was no significant difference in body weights between groups $(p>0.05)$. But according to an ingroup analysis, sitagliptin treated groups did not gain weight while non-treated groups gained weight significantly, between 14 th28th days.

The vasoactive responses of the thoracic aorta

Chronic NOS inhibition significantly increased contractile responses. Even though the dose-dependent curve of Phe was shifted to the left in the L-NAME group, the maximal force $\left(\mathrm{E}_{\mathrm{max}}\right)$ of the contraction was not different from other groups. On the other hand, there were no contractile responses in the first 3 doses of Phe, in the sitagliptin administered groups. Sitagliptin decreased the contractile responses of the HT+SITA group significantly with respect to the hypertensive group $(\mathrm{p}<0.05)$ (Fig. 3). $\mathrm{EC}_{50}$ value was significantly higher in the hypertensive group. This means the hypertensive group reached half of the maximum contraction with the lowest Phe concentration. $-\log \mathrm{EC}_{50}$ value of the HT+SITA group was significantly lower than in the hypertensive group; it demonstrates that reaching half of the maximum contraction needed higher concentration. Sitagliptin reduced the contraction responses $(\mathrm{p}<0.05)$ (Tab. 2).

Surprisingly there was a significant improvement in vasorelaxation due to $\mathrm{ACh}$ in only one concentration (Fig. 4a). Maximal relaxant responses between groups did not reach significance but $\mathrm{ACh} \mathrm{EC}_{50}$ values of the hypertensive group were significantly different from all groups. Sitagliptin treatment adducted the $-\log \mathrm{EC}_{50}$ values of the HT+SITA group to the control $(p<0.05)$ 

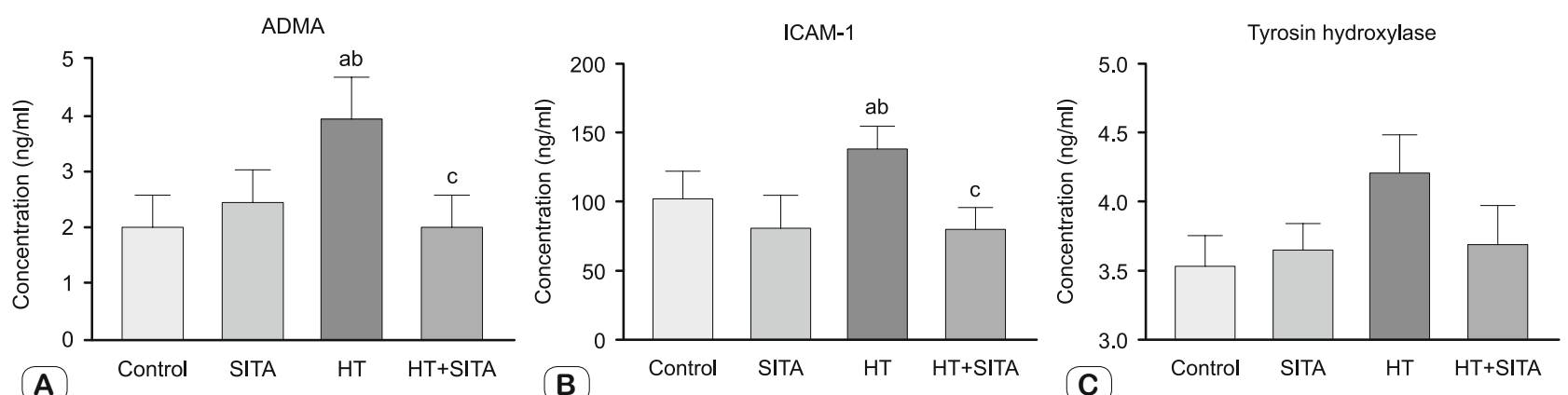

Fig. 5. Serum levels of asymmetric dimethylarginine (ADMA) (A), intercellular cell adhesion molecule (ICAM-1) (B) and Tyrosine hydroxylase (TH) (C). $n=7$, for each group. Data are expressed as the mean \pm S.E.M. a. $p<0.05$ compared to control, b. $p<0.05$ compared to SITA, c. $p<0.05$ compared to HT. SITA - sitagliptin; HT - hypertension.

(Tab. 2). Endothelium independent vasorelaxation by SNP was not different between groups ( $\mathrm{p}>0.05)$ (Fig. $4 \mathrm{~b})$.

\section{Serum ADMA, ICAM-1 and TH levels}

The level of ADMA increased from $2.02 \pm 0.56 \mathrm{ng} / \mathrm{ml}$ in the control group to $3.96 \pm 0.71 \mathrm{ng} / \mathrm{ml}$ in hypertensives. Serum ICAM-1 levels raised to $138.19 \pm 16.75 \mathrm{ng} / \mathrm{ml}$ in the HT group, significantly. Sitagliptin treatment decreased ADMA and ICAM-1 levels, significantly $(\mathrm{p}<0.05)$ (Figs 5a, 5b). TH increased approximately by $14 \%$, from $3.71 \mathrm{ng} / \mathrm{ml}$ (in controls) to $4.212 \mathrm{ng} / \mathrm{ml}$ (in HT group). Although sitagliptin reduced TH levels in hypertensive rats, it was not significant $(\mathrm{p}>0.05)$ (Fig. 5c).

\section{miRNA-21 and miRNA-155 expressions}

MicroRNA-21 expression of HT group increased $~ 3.3$ times compared to control and decreased 4.7 times with sitagliptin treatment. While miRNA-155 expression increased 10-fold in the HT group, it decreased 2.76-fold with sitagliptin administration $(\mathrm{p}<$ 0.05) (Fig. 6).

\section{Discussion}

The current study demonstrated that sitagliptin succeeded in ameliorating BP via improving vascular responses in the pharmacologically induced hypertension model. Consistently, sitagliptin decreased the previously unexplored parameters; ADMA, ICAM-1, TH and especially miRNAs. HT correlated miRNA-155 and miRNA-21 fold changes decreased with sitagliptin treatment, which is the major novel finding.

Environmentally, genetically, renal, or pharmacologically induced (with DOCA+ salt or L-NAME ) models, are used to form an experimental HT. When it is not possible to reach spontaneous hypertensive rats, L-NAME is generally considered as a perfect agent for forming HT. This study is based on pharmacologically induced HT. L-NAME at a dose of $40 \mathrm{mg} / \mathrm{kg}$ /day increased SBP significantly, as in a previous study (5).

As is known to all, sitagliptin is a very successful and advantageous widely used medication in type-2 DM treatment since it acts in the presence of glucose and does not cause hypoglycemia

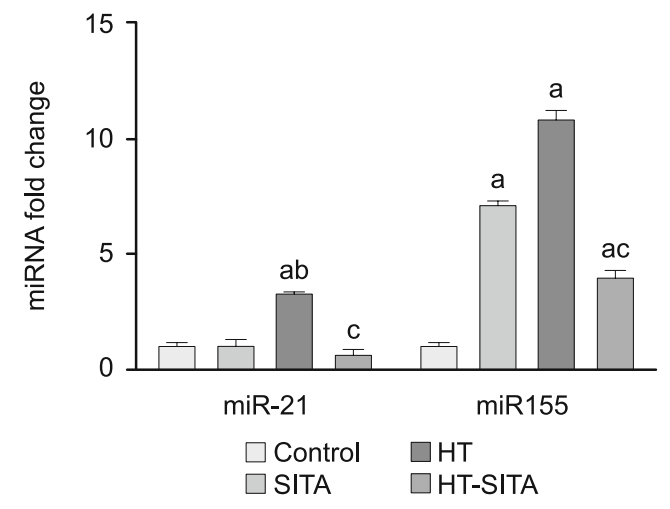

Fig. 6. Demonstration of fold changes of aortic tissue miRNA-21 and miRNA-155 with sitagliptin (qRT-PCR). Data are presented as mean \pm S.E.M, and $n=7$ for each group of the experiment. a. $p<0.05$ compared to control, b. $p<0.05$ compared to SITA, c. $p<0.05$ compared to HT. SITA - sitagliptin; HT - hypertension.

(27). Many researchers mentioned that sitagliptin has some pleiotropic properties like attenuating BP, lipid profile and body weight $(28,29)$. In this study, body weights were analyzed. Although no significant difference was found between groups, ingroup analysis showed that hypertensives and controls have put on weight significantly. It can be interpreted as an ordinary outcome of HT due to oedema for hypertensives. However, this comment does not explain the suppression of weight gain with sitagliptin and the continuation of weight gain in the control group. As Lanfranco D'Elia et al. (6) stated in their article, excess body weight may predispose to HT. According to this knowledge in our study, it can be said that sitagliptin also contributes to attenuation of BP by body weight control.

Blood pressure alleviation by sitagliptin has been reported in both, clinical studies and experimental models, in the presence or absence of concomitant DM $(31,32)$. Especially the experimental protocols contain different drug application duration, distinct sitagliptin dosage and different kinds and genetics of animals. As a result, the effect on BP (systolic, diastolic or mean) was controversial. Although Oliveira et al (33) used a dose of $200 \mathrm{mg} / \mathrm{kg}$ sitagliptin twice daily for seven days, we preferred to give sitagliptin in a dose of $80 \mathrm{mg} / \mathrm{kg} /$ day by oral gavage, as consistent 
with Giannaco et al (24), in this study. This reference study concluded that this dose suppresses the DPP-4 activity by $85 \%$ without hypoglycemia. Our measurements were mounting this conclusion; sitagliptin decreased the SBP significantly in the treatment group. On the other hand, sitagliptin lowered BP minimally in only sitagliptin applied group compared to normotensives but it did not reach significance. This can be interpreted as sitagliptin has an advantage that is effective in the case of pathologies like inflammation or endothelial dysfunction seen in hypertension.

Endothelial dysfunction is considered an early crucial event in the process of HT and cardiovascular diseases that is the main cause of death in diabetic patients. Since NO bioavailability and endothelium-mediated vasorelaxation are the hallmarks of the proper endothelial function, most studies are based on this information $(34,35)$. According to most preclinical researches, sitagliptin ameliorates impaired endothelial function with one of the probable mechanisms which is enhancement of the plasma concentration of GLP-1 and NO release (31). And thus, sitagliptin ensures endothelium-dependent relaxation of the aorta in response to ACh (25). Consistent with the literature, ACh responses in a cumulative manner and $\mathrm{EC}_{50}$ values showing the dose that supplies half of the maximum relaxation, confirmed that sitagliptin increased vasorelaxation. The reason why sitagliptin does not show its vasodilatory effect in all concentrations may be due to the use of L-NAME. While sitagliptin increased NO that dilates vessels, L-NAME decreased the NO with NOS inhibition. On the other hand, although it was not significant, the $\mathrm{ACh}$ responses and $\mathrm{E}_{\max }$ values were bigger than in the controls. These results can be interpreted again like, sitagliptin may affect healthy endothelium, but the main protective effect is supplied in case of pathology. Endothelium-independent vasodilatation was observed with SNP as in the study of Nade SV et al (36). In the work of Tang ST et al (25) both, endothelium-dependent and independent responses were evaluated. Another experiment showed relaxation responses to SNP in a different kind of animal (a rabbit) (37). In these studies, SNP did not change relaxation responses and the current study's findings are consistent with them. In the light of all these in-vitro results, vasorelaxation of aortas may be enunciated to be endothelium-dependent and sitagliptin shows its BP decreasing effect via NO-dependent mechanisms.

The endothelium protective effect of sitagliptin was also evaluated with ADMA at the molecular level in the study. It has been well established already before, endothelial dysfunction marker ADMA is an important risk factor for $\mathrm{HT}(38,39)$. Dimethyl aminohydrolase (DDAH) is the degradation enzyme for ADMA. Genetically DDAH deficient mice had increased serum ADMA levels and BP, besides decreased eNOS and ACh relaxation response (38). To the best of our knowledge, on the molecular level, there is no study yet that investigates serum ADMA concentrations with sitagliptin treatment. In the current study, ADMA increased in HT in compliance with the literature. That sitagliptin treatment decreased ADMA levels significantly in hypertensive rats was reported for the first time by this research. As mentioned before it can be considered that sitagliptin may improve endothelial dysfunction and protects vascular tone.
The sympathetic nervous system plays a critical role in the moment to moment control of BP due to either decreased betaadrenergic vasorelaxation or increased alfa-adrenergic sensitivity. It has been stated before that TH increased significantly in a hypertensive model (40). But the current study is the first that evaluates the effect of sitagliptin on TH. As there are limitations of our work ( maybe because of the low number of animal or the short duration of the study), the results did not reach significance. However, the tendency of TH levels to decrease with sitagliptin in the treatment group can be evaluated in later studies.

Chronic stimulation of the adrenergic system induces vascular dysfunction which is associated with increased inflammatory cytokines production. Chronic low-grade inflammation contributes to HT, especially by damaging the structure of the vessels. Inflammatory parameters in different kinds of pathologies and the effect of sitagliptin on these processes are tested generally. In a liver cancer study, sitagliptin decreased IL-6, IL-1 $\beta$ and TNF- $\alpha$ levels (41). In a clinical study comparing sitagliptin with another oral antidiabetic in type-2 DM, sitagliptin has been reported to significantly reduced CRP levels much more effectively (42). For instance, it was indicated that sitagliptin reduced inflammatory cell infiltration in unilaterally nephrectomized nondiabetic hypertensive rats (43). According to our knowledge, there was no investigation about the effect of sitagliptin on ICAM-1 levels in the experimental model of HT. Our study revealed that sitagliptin can decrease ICAM-1 effectively in HT almost as much as in the control group. One of the implications of our work is that sitagliptin may be more active in any pathological condition like endothelial dysfunction. The clinical study of Tremblay et al (14), supports our hypothesis. They found that inflammatory markers were higher in accompanying disease as expected. But the remarkable finding that matches our results is, sitagliptin is more active in patients with high inflammatory parameters.

After the genetic activity of miRNAs is recognized in recent years, their relationship with chronic diseases is still being tried to be defined. To determine the effect of sitagliptin on gene-level, we chose miRNA-155 and miRNA-21 that show a positive correlation in endothelial dysfunction and inflammation. Upregulated miRNA-155 and miRNA-21 results were correlated with ADMA, in vitro and ICAM in hypertensives and downregulation was applied in treated groups. To our knowledge, in the current study, we provided evidence that two miRNAs are good targets for sitagliptin firstly. On the other hand, we do not presently understand why miRNA-155 in only the sitagliptin applied group increased differently from the control group. It can be interpreted as; we know sitagliptin has many pleiotropic properties and affects different pathways, but perhaps what we do not know yet is that sitagliptin should not be used without any pathology.

For nearly a year, the whole world is fighting COVID-19. HT and $\mathrm{DM}$ are the most common accompanying diseases in people with severe COVID-19 and most of the pathologies of severe COVID-19 are also the main problems in DM and HT $(15,44)$. The core pathology of coronavirus disease is an infection of airway cells and endothelial dysfunction and thrombocytopathy are the essential components of severe disease (45). Also, according to what Porzionato et al mentioned, sympathetic activation is a 
potential link between comorbidities and COVID-19. With a new pharmacological approach, sitagliptin seems to be a miraculous drug that can suppress certain inflammatory markers, attenuate endothelial dysfunction and inappropriate sympathetic activity in COVID-19, accompanied by comorbidities (16). Sitagliptin seems to be even more important in critical disease processes with its main and pleiotropic characteristics.

\section{Conclusion}

The current study has confirmed that sitagliptin attenuates endothelial dysfunction and decreases inappropriate sympathetic activity and inflammation. At the same time, sitagliptin also downregulated two miRNAs which may become good biomarkers and treatment targets for HT. In summary, sitagliptin may control comorbidities (especially hypertension) of diabetic patients and introduces new targets to promote vascular responses.

\section{References}

1. Haldar R. A global brief on Hypertension: Silent killer, global public health crisis. IJOPMR 2013; 24 (1): 2-2.

2. Brook RD, Appel LJ, Rubenfire M et al. Beyond medications and diet: alternative approaches to lowering blood pressure: a scientific statement from the american heart association. Hypertension 2013; 61 (6): $1360-1383$.

3. Pawaskar M, Liu J, Rajpathak S, Iglay K, Engel SS, Hannachi H. Demographic and clinical profiles of type 2 diabetes mellitus patients initiating sitagliptin in the real-world setting. Curr Med Res Opin 2017; 33 (7): 1247-1253.

4. James PA, Oparil S, Carter BL et al. 2014 evidence-based guideline for the management of high blood pressure in adults: report from the panel members appointed to the Eighth Joint National Committee (JNC 8) [published correction appears in JAMA 2014 May 7; 311 (17): 1809]. JAMA 2014; 311 (5): 507-520.

5. Wong WT, Wong SL, Tian XY, Huang Y. Endothelial dysfunction: the common consequence in diabetes and hypertension. J Cardiovasc Pharmacol 2010; 55 (4): 300-307.

6. Action to Control Cardiovascular Risk in Diabetes Study Group, Gerstein HC, Miller ME et al. Effects of intensive glucose lowering in type 2 diabetes. N Engl J Med 2008; 358 (24): 2545-2559.

7. Munir KM, Lamos EM. Diabetes type 2 management: what are the differences between DPP-4 inhibitors and how do you choose?. Expert Opin Pharmacother 2017; 18 (9): 839-841.

8. Zhong J, Rajagopalan S. Dipeptidyl Peptidase-4 Regulation of SDF1/CXCR4 Axis: Implications for Cardiovascular Disease. Front Immunol 2015; 6:477.

9. Al-Awar A, Almási N, Szabó R et al. Novel Potentials of the DPP-4 Inhibitor Sitagliptin against Ischemia-Reperfusion (I/R) Injury in Rat ExVivo Heart Model. Int J Mol Sci 2018; 19 (10): 3226.

10. Dogan I, Dogan T, Yetim M et al. Relation of Serum ADMA, Apelin-13 and LOX-1 Levels with Inflammatory and Echocardiographic Parameters in Hemodialysis Patients. Ther Apher Dial 2018; 22 (2): 109-117.

11. Tayebati SK, Tomassoni D, Di Cesare Mannelli L, Amenta F. Effect of treatment with the antioxidant alpha-lipoic (thioctic) acid on heart and kidney microvasculature in spontaneously hypertensive rats. Clin Exp Hypertens 2016; 38 (1): 30-38.

12. Lang PP, Bai J, Zhang YL et al. Blockade of intercellular adhesion molecule-1 prevents angiotensin II-induced hypertension and vascular dysfunction. Lab Invest 2020; 100 (3): 378-386.

13. Esposito G, Cappetta D, Russo R et al. Sitagliptin reduces inflammation, fibrosis and preserves diastolic function in a rat model of heart failure with preserved ejection fraction [published correction appears in Br J Pharmacol 2018 May; 175 (10 ): 1781]. Br J Pharmacol 2017; 174 (22): 4070-4086.

14. Tremblay AJ, Lamarche B, Deacon CF, Weisnagel SJ, Couture P. Effects of sitagliptin therapy on markers of low-grade inflammation and cell adhesion molecules in patients with type 2 diabetes. Metabolism 2014; 63 (9): 1141-1148.

15. Fang L, Karakiulakis G, Roth M. Are patients with hypertension and diabetes mellitus at increased risk for COVID-19 infection? [published correction appears in Lancet Respir Med 2020 Jun; 8 (6): e54]. Lancet Respir Med 2020; 8 (4): e21.

16. Solerte SB, D'Addio F, Trevisan R et al. Sitagliptin Treatment at the Time of Hospitalization Was Associated With Reduced Mortality in Patients With Type 2 Diabetes and COVID-19: A Multicenter, Case-Control, Retrospective, Observational Study. Diabetes Care 2020; 43 (12): 2999-3006.

17. Dastan F, Abedini A, Shahabi S, Kiani A, Saffaei A, Zare A. Sitagliptin Repositioning in SARS-CoV-2: Effects on ACE-2, CD-26, and Inflammatory Cytokine Storms in the Lung. Iran J Allergy Asthma Immunol 2020; 19 (S1): 10-12.

18. Kozomara A, Griffiths-Jones S. miRBase: annotating high confidence microRNAs using deep sequencing data. Nucleic Acids Res 2014; 42 (Database issue): D68-D73.

19. Sun HX, Zeng DY, Li RT et al. Essential role of microRNA-155 in regulating endothelium-dependent vasorelaxation by targeting endothelial nitric oxide synthase. Hypertension 2012; 60 (6): 1407-1414.

20. Mann M, Mehta A, Zhao JL et al. An NF- $\kappa B$-microRNA regulatory network tunes macrophage inflammatory responses [published correction appears in Nat Commun 2018 Aug 16; 9 (1): 3338]. Nat Commun 2017; 8 (1): 851.

21. Kontaraki JE, Marketou ME, Parthenakis FI et al. Hypertrophic and antihypertrophic microRNA levels in peripheral blood mononuclear cells and their relationship to left ventricular hypertrophy in patients with essential hypertension. J Am Soc Hypertens 2015; 9 (10): 802-810.

22. Gangwar RS, Rajagopalan S, Natarajan R, Deiuliis JA. Noncoding RNAs in Cardiovascular Disease: Pathological Relevance and Emerging Role as Biomarkers and Therapeutics. Am J Hypertens 2018; 31 (2): $150-165$.

23. Li X, Wei Y, Wang Z. microRNA-21 and hypertension. Hypertens Res 2018; 41 (9): 649-661.

24. Giannocco G, Oliveira KC, Crajoinas RO et al. Dipeptidyl peptidase IV inhibition upregulates GLUT4 translocation and expression in heart and skeletal muscle of spontaneously hypertensive rats. Eur J Pharmacol 2013; 698 (1-3): 74-86.

25. Tang ST, Su H, Zhang Q et al. Sitagliptin inhibits endothelin-1 expression in the aortic endothelium of rats with streptozotocin-induced diabetes by suppressing the nuclear factor- $\mathrm{\kappa B} / \mathrm{I} \kappa \mathrm{B} \alpha$ system through the activation of AMP-activated protein kinase. Int J Mol Med 2016; 37 (6): $1558-1566$. 
26. Simko F, Baka T, Poglitsch M et al. Effect of Ivabradine on a Hypertensive Heart and the Renin-Angiotensin-Aldosterone System in L-NAMEInduced Hypertension. Int J Mol Sci 2018; 19 (10): 3017.

27. Scott LJ. Sitagliptin: A Review in Type 2 Diabetes. Drugs 2017; 77 (2): 209-224.

28. Hussain M, Atif MA, Tunio AG, Ali B, Akhtar L, Serwar G. Effect Of Sitagliptin On Glycemic Control, Body Weight, Blood Pressure And Serum Lipid Profile In Type 2 Diabetic Hyperlipidemic Patients. J Ayub Med Coll Abbottabad 2016; 28 (2): 369-372.

29. Zhou Y, Guo Z, Yan W, Wang W. Cardiovascular effects of sitagliptin - An anti-diabetes medicine. Clin Exp Pharmacol Physiol 2018; 45 (7): 628-635.

30. D'Elia L, Strazzullo P. Excess Body Weight, Insulin Resistance and Isolated Systolic Hypertension: Potential Pathophysiological Links. High Blood Press Cardiovasc Prev 2018; 25 (1): 17-23.

31. Liu L, Liu J, Wong WT et al. Dipeptidyl peptidase 4 inhibitor sitagliptin protects endothelial function in hypertension through a glucagon-like peptide 1-dependent mechanism. Hypertension 2012; 60 (3): 833-841.

32. Duvnjak L, Blaslov K. Dipeptidyl peptidase-4 inhibitors improve arterial stiffness, blood pressure, lipid profile and inflammation parameters in patients with type 2 diabetes mellitus. Diabetol Metab Syndr 2016; 8: 26 .

33. Oliveira BC, Marques VB, Brun BF et al. Dipeptidyl peptidase-4 inhibition prevents vascular dysfunction induced by $\beta$-adrenergic hyperactivity. Biomed Pharmacother 2019; 113:108733.

34. Taddei S, Ghiadoni L, Virdis A, Versari D, Salvetti A. Mechanisms of endothelial dysfunction: clinical significance and preventive nonpharmacological therapeutic strategies. Curr Pharm Des 2003; 9 (29): 2385-2402.

35. Sena CM, Pereira AM, Seiça R. Endothelial dysfunction - a major mediator of diabetic vascular disease. Biochim Biophys Acta 2013; 1832 (12): 2216-2231.
36. Nade VS, Kawale LA, Patel KM. Protective effect of sitagliptin and rosuvastatin combination on vascular endothelial dysfunction in type-2 diabetes. Indian J Pharm Sci 2015; 77 (1): 96-102.

37. Nader MA. Sitagliptin ameliorates lipid profile changes and endothelium dysfunction induced by atherogenic diet in rabbits. Naunyn Schmiedebergs Arch Pharmacol 2014; 387 (5): 433-444.

38. Hu X, Xu X, Zhu G et al. Vascular endothelial-specific dimethylarginine dimethylaminohydrolase-1-deficient mice reveal that vascular endothelium plays an important role in removing asymmetric dimethylarginine. Circulation 2009; 120 (22): 2222-2229.

39. Wang Y, Dong X. Nebivolol ameliorates asymmetric dimethylarginine-induced vascular response in rat aorta via $\beta 3$ adrenoceptor-mediated mechanism. Clin Exp Hypertens 2016; 38 (2): 252-259.

40. Sahna E, Kurcer Z, Ozturk F et al. Effects of chronic ethanol consumption on alpha-adrenergic-induced contractions and endothelium-dependent relaxations in rat thoracic aorta. Pharmacol Res 2000; 41 (6): 629-633.

41. Jiang W, Wen D, Cheng Z, Yang Y, Zheng G, Yin F. Effect of sitagliptin, a DPP-4 inhibitor, against DENA-induced liver cancer in rats mediated via NF- $\mathrm{KB}$ activation and inflammatory cytokines. J Biochem Mol Toxicol 2018; 32 (12): e22220.

42. Sun Y, Yan D, Hao Z, Cui L, Li G. Effects of Dapagliflozin and Sitagliptin on Insulin Resistant and Body Fat Distribution in Newly Diagnosed Type 2 Diabetic Patients. Med Sci Monit 2020; 26:e921891. DOI:10.12659/ MSM.921891

43. Jo CH, Kim S, Park JS, Kim GH. Anti-Inflammatory Action of Sitagliptin and Linagliptin in Doxorubicin Nephropathy. Kidney Blood Press Res 2018; 43 (3): 987-999.

44. Cuschieri S, Grech S. COVID-19 and diabetes: The why, the what and the how. J Diabetes Complications 2020; 34 (9): 107637.

45. Gu SX, Tyagi T, Jain K et al. Thrombocytopathy and endotheliopathy: crucial contributors to COVID-19 thromboinflammation. Nat Rev Cardiol 2021; 18 (3): 194-209. 